3-ORIGINAL ARTICLE

TECHNICAL SKILL

\title{
Modified "Roux en Y" hepaticojejunostomy to permit transgastric endoscopic access ${ }^{1}$
}

\author{
André de Moricz, Otavio Schimdt Azevedo ${ }^{\mathrm{II}}$, Tércio De Campos ${ }^{\mathrm{III}}$, Rogério Colaiacovo ${ }^{\mathrm{IV}}$, Tadayoshi Akiba ${ }^{\mathrm{V}}$, Rodrigo Altenfelder \\ Silva ${ }^{\mathrm{VI}}$, Adhemar Monteiro Pacheco JrVII.
}

DOI: http://dx.doi.org/10.1590/S0102-86502014001700003

${ }^{\mathrm{I}} \mathrm{PhD}$, Assistant Professor, Department of Surgery, Faculty of Medical Sciences, Santa Casa School of Medicine, São Paulo, Brazil. Acquisition and interpretation of data, technical procedures, manuscript writing, critical revision.

IIPost-graduate student, Department of Surgery, Faculty of Medical Sciences, Santa Casa School of Medicine, São Paulo, Brazil. Acquisition and interpretation of data, technical procedures, manuscript preparation,

IIIPhD, Associate Professor, Department of Surgery, Faculty of Medical Sciences, Santa Casa School of Medicine, São Paulo, Brazil. Acquisition and interpretation of data, technical procedures.

${ }^{\mathrm{IV}}$ Instructor Department of Peroral Endoscopy, Faculty of Medical Sciences, Santa Casa School of Medicine, São Paulo, Brazil. Analysis and interpretation of data, technical procedures.

${ }^{\mathrm{v}}$ First Assistant, Department of Peroral Endoscopy, Faculty of Medical Sciences, Santa Casa School of Medicine, São Paulo, Brazil. Technical procedures. ${ }^{V} \mathrm{PhD}$, Associate Professor, Department of Surgery, Faculty of Medical Sciences, Santa Casa School of Medicine, São Paulo, Brazil. Conception and design of the study, technical procedures, critical revision.

VIIPhD, Associate Professor, Department of Surgery, Faculty of Medical Sciences, Santa Casa School of Medicine, São Paulo, Brazil. Conception and design of the study, technical procedures, critical revision.

\begin{abstract}
PURPOSE: To present an option technique during the accomplishment of a "Roux-en-Y" hepaticojejunostomy that, allows future transgastric endoscopic access to the biloenteric anastomosis.

METHODS: The side-to-side "Roux-en-Y" hepaticojejunostomy is carried through transmesocolic, retrogastric short tunnel. A distal jejunal loop of approximately 10,0 to 15,0 cm must be kept far from the anastomosis that allows its setting, without tension, in the anterior gastric wall bellow the small bending, between body and of the stomach antrum. Once the jejunal segment is fixed on the anterior gastric wall, this will allow future endoscopic access to the bilioenteric anastomosis, carried through transgastric punction guided by endoscopic-ultrasound (EUS).
\end{abstract}

CONCLUSION: The modified "Roux en Y" hepaticojejunostomy is a simple and new technique to permit an alternative transgastric endoscopic access to bilioenteric anastomosis.

Key words: Biliary anastomosis, surgical technique, endoscopy. 


\section{Introduction}

There are several indications for performing "Rouxen-Y" hepaticojejunostomy such as, bile duct injury, malignant strictures, benign strictures, multiple gallstones and liver transplantation ${ }^{1}$. Once performed, it can develop complications such as postoperative bile duct strictures, intra hepatic gallstones and chronic cholestasis. The outcome of patients if not treated will be acute cholangitis, hepatic fibrosis, and in more advanced cases secondary biliary cirrhosis and liver failure ${ }^{2,3}$

Therapeutic options involve reoperation of the biliary tract, endoscopic procedures or interventional radiology, often difficult due to intrahepatic multiple surgical anastomoses ${ }^{4}$. To facilitate access to the biliary tract in cases of recurrence of stenosis the strap to the hepaticojejunostomy can be attached in the abdominal wall to permit further endoscopic approach to the biliary-enteric anastomosis. Also described is the double balloon endoscopy or, an alternative endoscopic approach such as, video-assisted laparoscopy ${ }^{4,5}$. Another option is an interventional radiology with trans-hepatic access to the biliary tract ${ }^{6,7}$.

Many of these procedures depend on the logistics of advanced radiology, generate discomfort to the patient in cases of ostomy or rely on more invasive and repeated biliary surgical procedures $^{4,5,6,7}$.

The objective of this work is to, describe an optional technique during the accomplishment of a "Roux-en-Y" hepaticojejunostomy that, allows future transgastric endoscopic access to the biliary-enteric anastomosis.

\section{Methods \\ Surgical technique}

The side-to-side "Roux-en-Y" hepaticojejunostomy is carried through transmesocolic, retrogastric short tunnel. A distal jejunal loop of approximately 10,0 to $15,0 \mathrm{~cm}$ must be kept far from the anastomosis that allows its setting, without tension, in the anterior gastric wall bellow the small bending, between body and the stomach antrum (Figure 1).

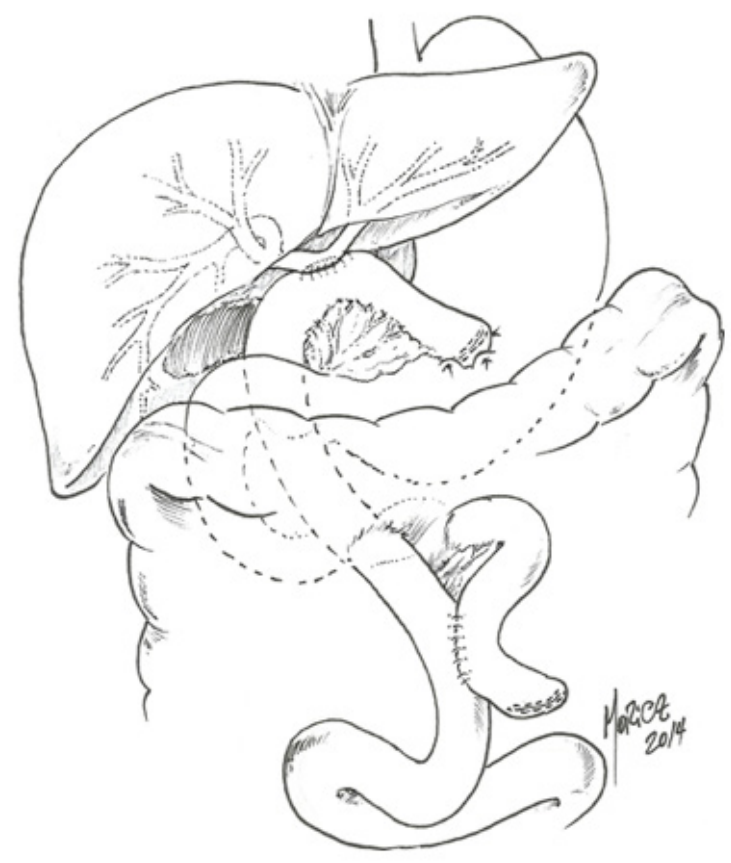

FIGURE 1. Modified hepaticojejunostomy.

Once the jejunal segment is fixed on the anterior gastric wall, this will allow future endoscopic access to the bilioenteric anastomosis, carried through transgastric punction guided by endoscopic-ultrasound (EUS) which facilitates identification of the strap attached to the gastric body (Figure 2).

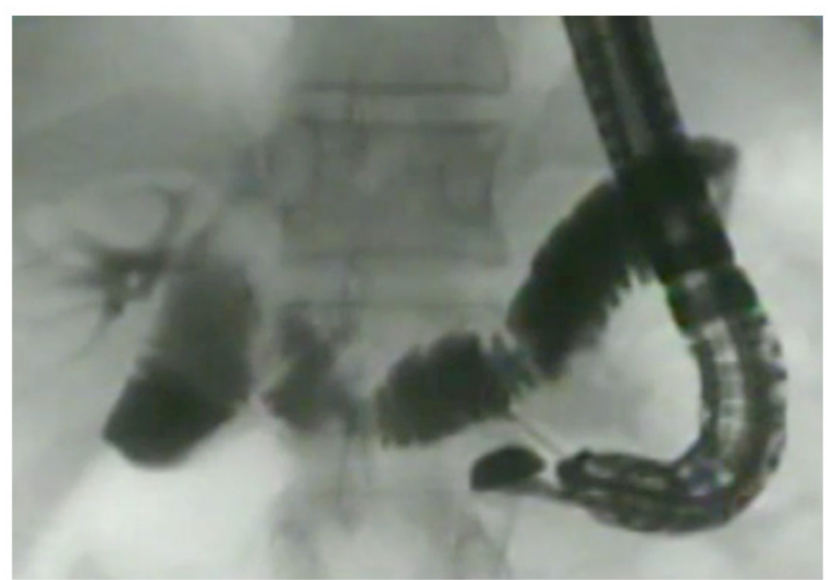

FIGURE 2 - Transgastric EUS access to hepaticojejunostomy.

\section{Discussion}

Patients who develop complications of hepaticojejunostomies such as anastomotic stenosis are difficult to treat. The recurrent bouts of cholangitis due to chronic cholestasis and intrahepatic stones are the main cause of progressive loss of liver function. In these cases the performance of a new biliary- 
enteric anastomosis is the most widely used therapeutic option. However, the high anastomosis in hilar and intrahepatic board, have high morbidity and mortality, with complication rates of up to $43 \%{ }^{2}$. Recurrence of stenosis is common in about $25 \%$ of cases and will require further intervention. Two-thirds of recurrences occur within two years and $90 \%$ within five years ${ }^{8,9}$.

Less invasive treatment options that can be performed repeatedly are of great value in the complications of "Rouxen-Y" hepatojejunostomies. Currently among these options have interventional radiology and endoscopic therapies have been used more frequently. Through these you can unclog and wash the bile, dilate stenosis and pass transanastomotic stents to treat and prevent cholestasis and further hepatic dysfunction ${ }^{10,11}$.

While through the trans-hepatic approach there are complications related to puncture and permanence of external catheters, in patients with "Roux-en-Y", the endoscopic access to the anastomosis is hampered by the distance traveled by the jejunal loop until reaching the angle of the enteral anastomosis ${ }^{5,6,7}$. To overcome such problems, innovative endoscopic techniques such as double balloon endoscopy, fixing the jejunal loop of the hepaticojenunostomy in the abdominal wall, invasive laparoscopic video-assisted endoscopic procedures are described. The best approach is selected on a case by case basis ${ }^{2,8,9}$.

In this context we propose an alternative approach for accessing the "Roux-en-Y" hepaticojejunostomies that can be performed by ultrasound endoscopy. This simple technique has the advantages related to the absence of ostomies and external catheters, permit repeated endoscopic procedures to access the anastomotic problems with shortened distances through the jejunal loop.

\section{Conclusion}

The modified "Roux en Y" hepatojejunostomy is a simple and new technique to permit an alternative transgastric endoscopic access to biliary-enteric anastomosis.

\section{References}

1. Dolan JP, Diggs BS, Sheppard BC, Hunter JG. Ten-year trend in the national volume of bile duct injuries requiring operative repair. Surg Endosc. 2005; 19:967.

2. Lillemoe KD, Melton GB, Cameron JL, Pitt HA, Campbell KA, talamini MA, Sauter PA, Coleman J Yeo CJ. Postoperative bile duct strictures: management and outcome in the 1990s. Ann Surg. 2000; 232:430.
3. Jabłońska B, Lampe P, Olakowski M, Górka Z, Lekstan A, Gruszka T. Hepaticojejunostomy vs. end-to-end biliary reconstructions in the treatment of iatrogenic bile duct injuries. J Gastrointest Surg. 2009; 13:1084.

4. Hintze RE, Adler A, Veltzke W, Abou-Rebyeh H. Endoscopic access to the papilla of Vater for endoscopic retrograde cholangiopancreatography in patients with Billroth II or Roux-en-Y gastrojejunostomy. Endoscopy. 1997; 29:69.

5. Lopes TL, Wilcox CM. Endoscopic retrograde cholangiopancreatography in patients with Roux-en-Y anatomy. Gastroenterol Clin North Am. 2010; 39:99.

6. Pessa ME; Hawkins IF \& Vogel SB. The treatment of acute cholangitis: percutaneous transhepatic biliary drainage before definitive therapy. Ann Surg. 1987; 4: 389-392.

7. Chen M; Jan Y \& Lee T. Percutaneous transhepatic biliary drainage for acute cholangitis. Int Surg. 1987; 72: 131-133.

8. Sicklick JK, Camp MS, Lillemoe KD, Melton GB, Yeo CJ, Campbell KA, Talamini MA, Pitt HA, Coleman J, Sauter PA, Cameron JL. Surgical management of bile duct injuries sustained during laparoscopic cholecystectomy: perioperative results in 200 patients. Ann Surg. 2005; 241:786.

9. Walsh RM, Henderson JM, Vogt DP, Brown N. Long-term outcome of biliary reconstruction for bile duct injuries from laparoscopic cholecystectomies. Surgery. 2007; 142:450.

10. Ludwig K, Bernhardt J, Lorenz D. Value and consequences of routine intraoperative cholangiography during cholecystectomy. Surg Laparosc Endosc Percutan Tech. 2002; 12:154.

11. Parlak E, Ciçek B, Dişibeyaz S, Cengiz C, Yurdakul M, akdoan M, Kiliç MZY, Sasmaz N, Cumhur T, Sahin B. Endoscopic retrograde cholangiography by double balloon enteroscopy in patients with Roux-en-Y hepaticojejunostomy. Surg Endosc. 2010; 24:466.

\section{Correspondence:}

André Moricz

Rua Sincorá, 127

Jardim Umuarama, São Paulo - SP

CEP: 04650000

amoricz@uol.com.br

Tel: 11996149305

Tel: 1121170100

${ }^{1}$ Research performed at Department of Surgery, Biliary Tract and Pancreas Group, Endoscopic Ultrasound French-Brazilian Center, Faculty of Medical Sciences, Santa Casa School of Medicine, São Paulo, Brazil. 